\title{
Hipertensão Arterial na Infância e Adolescência: Definição e Investigação da Hipertensão Arterial
}

\author{
Maria Cristina de Andrade
}

\section{Resumo}

Cerca de 5\% das crianças e adolescentes tem hipertensão arterial, sendo que nas crianças, a hipertensão é mais comumente assintomática. A definição de hipertensão na infância baseia-se na distribuição normal da pressão arterial (PA) em crianças saudáveis.

O tamanho corporal é o determinante mais importante da PA em crianças e adolescentes. Desta forma, os percentis que normatizam os valores de PA são baseados em dados de medidas da pressão arterial de acordo com sexo, idade, altura em estudos de base populacional.

O objetivo desta apresentação é mostrar a técnica correta da mensuração da pressão arterial na infância e adolescência e os métodos de investigação de acordo com a provável etiologia: se primária ou secundária.

\section{Métodos:}

Definições de pressão arterial:

PA normal em crianças é definida como pressão arterial sistólica (PAS) e pressão arterial diastólica (PAD) inferior ao percentil 90 para idade, sexo e altura.

Pré-hipertensão: pressão sistólica e/ou diastólica percentil $\geq 90$ BP porém <percentil 95

Hipertensão: é definida como pressão sistólica e/ou diastólica percentil $\geq 95$ medido em três ou mais ocasiões.

Hipertensão estágio 1: PA sistólica e/ou diastólica entre o percentil 95 e 5 $\mathrm{mmHg}$ acima do percentil 99.

Hipertensão estágio 2: PA sistólica e/ou diastólica percentil $\geq 99$ BP mais 5 $\mathrm{mmHg}$. 
Discussão: O processo de decisão para a avaliação e tratamento varia de acordo com a gravidade da $\mathrm{HA}$.

O diagnóstico da HAS é dependente de medições precisas de rotina da pressão arterial durante a infância, através de um processo e equipamentos padronizados. Fatores de confusão incluem o tamanho do manguito, a técnica utilizada (isto é, da posição do paciente e da escolha do quarto ou quinto som Korotkoff para determinar diastólica), o número de medições efetuadas, e/ou o tipo de aparelho utilizado.

A avaliação inicial da criança com hipertensão arterial sistêmica (HAS) inclui anamnese, exame físico, exames laboratoriais e procedimentos. Com base na história inicial, no exame físico, e na avaliação laboratorial, o pediatra deve ser capaz de determinar se a hipertensão é primária (sem causa identificável) ou secundária ( quando uma doença de base é identificável). Esta distinção vai determinar se uma avaliação mais aprofundada deve ser realizada para se encontrar uma causa potencialmente reversível de HAS, como a HAS de origem reno-vascular. Resultados: A mensuração da pressão arterial é obrigatória a partir de 3 anos de idade nas consultas de rotina e nos atendimentos de emergência. Deve ser mensurada mais precocemente nos recém-nascidos em UTI neonatal, nos pacientes com cardiopatias congênitas, malformações do trato urinário ou anormalidades do exame de Urina I, assim como em situações com aumento da pressão intracraniana. Também é necessária se houver antecedente pessoal ou familiar de: doença renal, transplante de órgão sólido e/ou de medula, neoplasias, e uso de medicamentos que aumentam PA (beta2-estimulantes).

Conclusão: Atualmente está bem claro que a hipertensão (HAS) começa na infância e adolescência, e que contribui para o desenvolvimento precoce de doença cardiovascular (DCV).

Palavras chave: hipertensão arterial, infância, doença cardio-vascular.

De Andrade, Maria Cristina. "Hipertensão Arterial na Infância e Adolescência: Definição e Investigação da Hipertensão Arterial", in Anais do 20. Congresso Internacional Sabará de Especialidades Pediátricas 
Cerca de 5\% das crianças e adolescentes tem hipertensão arterial, e, em adultos, a hipertensão arterial é uma das principais causas de doença cardiovascular, doença renal em estágio terminal, e de acidente vascular cerebral. Apesar da lesão de órgãos-alvo decorrente da HAS ocorrer mais comumente em adultos, a hipertensão e suas complicações também ocorrem na infância. Nas crianças, a hipertensão é mais comumente assintomática, mas pode estar associada a mudanças sutis no comportamento ou no desempenho escolar.

As recomendações atuais estão resumidas em vários documentos, sendo que o mais utilizado é o quarto relatório sobre o diagnóstico, avaliação e tratamento da hipertensão em crianças e adolescentes (National High Blood Pressure Education Program Working Group (NHBPEP), the American Academy of Pediatrics, the American Heart Association Council on High Blood Pressure Research on routine BP measurements in children and adolescents, and the National Heart, Lung, and Blood Institute's Expert Panel on Integrated Guidelines).

A definição de hipertensão na infância baseia-se na distribuição normal da pressão arterial (PA) em crianças saudáveis. Isto está em contraste com a HAS adulto, que é definida principalmente por dados clínicos (ou seja, o risco de doença cardiovascular e mortalidade) obtidos de ensaios de terapia anti-hipertensiva.

O tamanho corporal é o determinante mais importante da PA em crianças e adolescentes. Assim, a classificação da PA é mais precisa quando os valores são ajustados para altura, bem como a idade e sexo para evitar interpretação inadequada de crianças nos extremos do crescimento normal. Desta forma, os percentis que normatizam os valores de PA são baseados em dados de medidas da pressão arterial de acordo com sexo, idade, altura em estudos de base populacional.

\section{Definições:}

PA normal em crianças é definida como pressão arterial sistólica (PAS) e pressão arterial diastólica (PAD) inferior ao percentil 90 para idade, sexo e altura. A PA sistólica e diastólica são de igual importância; e se houver uma disparidade entre as duas, o valor mais elevado determina a categoria da pressão arterial.

Pré-hipertensão: pressão sistólica e/ou diastólica percentil $\geq 90$ BP porém <percentil 95, ou em adolescentes, se a PA > 120X80 mmHg, mesmo abaixo do percentil 90. Globalmente, a prevalência de pré-hipertensão varia de 9,5 a $24 \%$ da população pediátrica. 
Hipertensão: é definida como pressão sistólica e/ou diastólica percentil $\geq 95$ medido em três ou mais ocasiões.

Hipertensão estágio 1: pressão sistólica e/ou diastólica entre o percentil 95 e 5 $\mathrm{mmHg}$ acima do percentil $99 \mathrm{ou}$, em adolescentes se a PA exceder 140X90 mmHg, mesmo < percentil 95.

Hipertensão estágio 2: sistólica e/ou diastólica percentil $\geq 99$ BP mais $5 \mathrm{mmHg}$.

O processo de decisão para a avaliação e tratamento varia de acordo com a gravidade da HA. Como observado acima, são definidas duas fases para HAS. Estágio 2 identifica as crianças que necessitam de uma avaliação mais rápida e tratamento farmacológico imediato, enquanto que o estágio 1 permite que haja mais tempo para avaliação e tratamento inicial com a terapia não farmacológica, a menos que o paciente seja sintomático ou apresente lesões de órgãos-alvo.

O diagnóstico da HAS é dependente de medições precisas de rotina da pressão arterial durante a infância, através de um processo e equipamentos padronizados. Fatores de confusão incluem o tamanho do manguito, a técnica utilizada (isto é, da posição do paciente e da escolha do quarto ou quinto som Korotkoff para determinar diastólica), o número de medições efetuadas, e/ou o tipo de aparelho utilizado.

Tamanho do manguito: Em relação à largura, a câmara de ar deve ocupar aproximadamente $40 \%$ perímetro do braço, que é medido a partir da determinação de um ponto médio entre o olécrano e o acrômio e 80 a 100\% em relação ao comprimento. Manguitos menores produzem leituras mais elevadas e manguitos maiores levam a resultados menores.

A medida da pressão arterial deve ser realizada no braço direito após o paciente ter ficado quieto e confortavelmente sentado por pelo menos três minutos em um ambiente tranquilo.

$\mathrm{Na}$ avaliação inicial, medidas de pressão arterial são obtidas em ambas as extremidades superiores e inferiores para detectar a possível presença de coarctação como a causa da hipertensão.

Número de medições - A PA deve ser tomada pelo menos duas vezes em cada consulta, com as medidas, separadas por um ou dois minutos para permitir a liberação de sangue. Se o segundo valor for maior que $5 \mathrm{~mm} \mathrm{Hg}$ diferente do primeiro, as medições devem ser continuadas até um valor estável seja atingido. $O$ valor registrado no prontuário do paciente deve ser a média das duas últimas medidas estáveis. 
Aparelho: Dispositivos oscilométricos são cada vez mais usados na prática devido à sua facilidade de utilização e por diminuir o viés do observador [47]. Eles são particularmente úteis quando ausculta é difícil como com bebês e recémnascidos, no ambiente de terapia intensiva, quando a medida da PA frequente é necessária, ou para mensuração inicial da PA em pronto-socorro movimentado.

No entanto, se a medida da pressão arterial for superior ao percentil 90 por oscilometria, ela deve ser confirmada por uma medida pelo método auscultatório.

Som: Ao realizar uma medição de PA pelo método auscultatório, o primeiro (surgimento do som) e quinto sons (desaparecimento) de Korotkoff são usados para identificar os valores pressão arterial sistólica e diastólica, respectivamente.

Todas as crianças maiores que três anos de idade devem ter sua pressão arterial medida nas consultas de rotina e nos atendimentos de emergência. Crianças com menos de três anos de idade devem ter sua pressão arterial aferida, se eles têm um histórico de internação na UTI neonatal, cardiopatias congênitas, malformações do trato urinário ou anormalidades do exame de Urina I, assim como também em situações com aumento da pressão intracraniana. Além disso, se houver antecedente pessoal ou familiar de: doença renal, transplante de órgão sólido e/ou de medula, neoplasias, e uso de medicamentos que aumentam PA (beta2estimulantes).

Os objetivos da avaliação inicial da criança e do adolescente hipertensos são:

Identificar hipertensão secundária (HTN) (isto é, uma causa subjacente da hipertensão), o que pode ser curada, evitando a necessidade de uma terapia medicamentosa prolongada.

$\square$ Identificar outros factores de risco (por exemplo obesidade e dislipidemia) para doença cardiovascular (DCV) ou doenças associadas com um risco aumentado de doenças cardiovasculares (por exemplo, diabetes mellitus)

$\square$ Identificar pacientes com estágio 2 HTN ou com evidência de lesão de órgãoalvo (retinopatia, hipertrofia de ventrículo esquerdo e proteinúria) para que a terapia farmacológica possa ser iniciada.

- A avaliação inicial da criança com hipertensão arterial sistêmica (HAS) inclui anamnese, exame físico e exames laboratoriais e procedimentos. Na HAS primária, normalmente a PA se contrada no Estágio 1( até P99 + $5 \mathrm{mmHg}$ ) e 
está associada a antecedentes familiares de HA, doença cardiovascular e obesidade.

A HAS no Estágio 2 (>P99 + 5 mmHg) apresenta risco maior de hipertensão secundária, sendo que quanto menor a idade e maior o nível pressórico, maior a probabilidade de HA secundária.

$\mathrm{Na}$ avaliação inicial, pesquisa-se na anamnese história positiva de: doença renal, cardíaca, outros órgãos. Também se pesquisa a presença de poliúria, polidípsia, distúrbios visuais, trauma, infecção do trato urinário, uso de medicações e suplementos. No exame físico deve-se realizar a avaliação crescimento e índice de massa corpórea (IMC), medição da pressão arterial nos quatro membros, fáscies, palpação de pulsos, fundo de olho, pesquisa de sopro abdominal e alteração de genitais, além de presença de púrpura, manchas café com leite e acantose nigrans. Avaliação laboratorial inicial: Função renal (uréia e creatinina), natremia e potassemia, hemograma, Urina I e Ultrassonografia renal.

Deve-se também realizar ecocardiograma (hipertrofia de ventrículo esquerdo), fundo de olho, glicemia de jejum, lipidograma, ácido úrico, microalbuminúria e hemoglobina glicosilada.

A investigação da hipertensão secundária deve ser a mesma da hipertensão estágio 1, acrescida de investigação direcionada para patologia de base, como por exemplo: avaliação da atividade da renina plasmática, pesquisa de HA renovascular por meio de angioRM, angioTC, US Doppler, Arteriografia; dosagens hormonais sérica e urinária de cortisol, aldosterona, catecolaminas.

Vale lembrar que as causas mais frequentes de HAS secundária são: renal 74 \%, coartação da aorta $15 \%$, doença renovascular $7 \%$, doenças da adrenal $1 \%$, doenças do SNC $1 \%$, doenças sistêmicas $1 \%$ e tumores extra-adrenais $1 \%$.

Além da classificação da hipertensão arterial em primária e secundária, existem:

Hipertensão do avental branco, que ser caracteriza por níveis de $\mathrm{PA}>\mathrm{P}_{95}$ no consultório médico ou clínica, que se normalizam fora do ambiente médico e deve ser avaliada pela monitorização da PA ambulatorial ou domiciliar (MAPA).

Hipertensão mascarada, que se caracteriza por PA normal no consultório e hipertensão na MAPA.

As indicações de MAPA são HA do avental branco, HA limítrofe ou instável, avaliação da terapêutica anti-hipertensiva, progressão de lesão em órgãos-alvo, doenças com risco de HÁ: 
diabetes mellitus, doença renal crônica e doença renal policística.

$\mathrm{Na}$ figura abaixo estão sumarizadas as etapas da investigação da hipertensão arterial na infância.

\section{Investigação da Hipertensão Arterial}

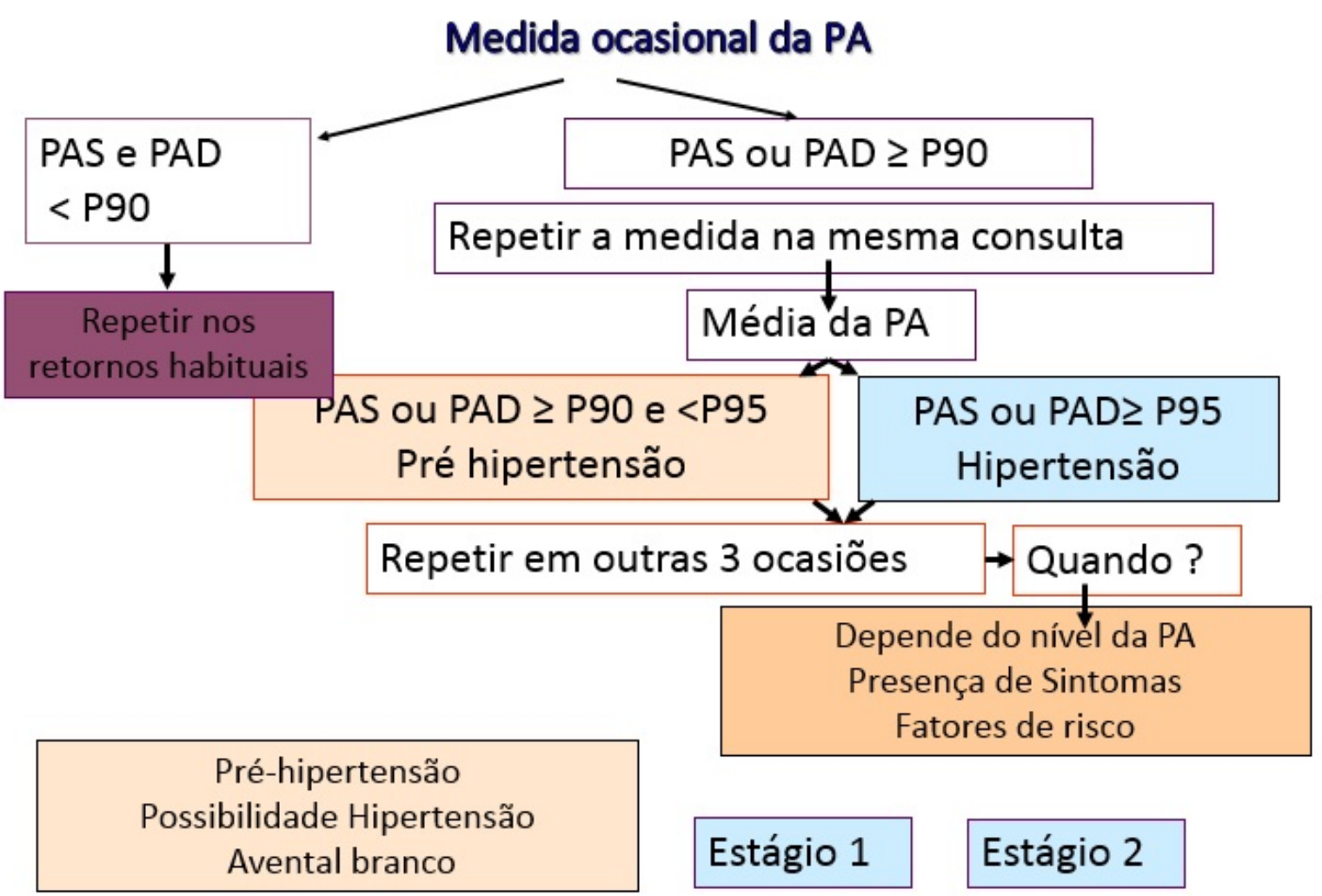

Hypertension in Children and Adolescents:

Definition and evaluation of hypertension

\section{Abstract:}

About $5 \%$ of children and adolescents have high blood pressure, and in children, hypertension is most commonly asymptomatic. The definition of hypertension in children is based on the normal distribution blood pressure (BP) in healthy children. The body size is the most important determinant of BP in children and adolescents. Thus, the percentiles that regulate $\mathrm{BP}$ values are based on data from blood pressure measurements according to sex, age, height in population-based studies. 
The purpose of this lecture is to show the correct technique of measuring blood pressure in childhood and adolescence and research methods according to the probable etiology: primary or secondary.

\section{Methods:}

Definitions of blood pressure:

Normal BP in children is defined as systolic and diastolic blood pressure (SBP and DBP) lower than the 90th percentile for age, sex and height .

Pre-hypertension: systolic and or diastolic BP percentile $\geq 90$ th but $<95$ th percentile Hypertension is defined as systolic and/or diastolic $\geq 95$ th percentile measured on three or more occasions pressure.

Stage 1 hypertension: systolic and/or diastolic blood pressure between the 95th and $5 \mathrm{mmHg}$ above the 99th percentile.

Stage 2 hypertension: systolic and/or diastolic BP percentile plus $5 \mathrm{mmHg} \geq 99$ th.

Discussion: The decision process for the evaluation and treatment will vary with the severity of hypertension.

The diagnosis of hypertension is dependent on accurate routine measurements of blood pressure during childhood through the process and standardized equipment. Confounding factors included the cuff size, the technique used (i.e. the position of the patient and the choice of the fourth or fifth Korotkoff sound to determine diastolic), the number of measurements taken and/ $r$ the type of device used.

The initial evaluation of the child with systemic arterial hypertension (SAH) includes history, physical examination and laboratory tests and procedures. Based on the initial history, physical examination, and laboratory evaluation, pediatrician should be able to determine if hypertension is primary (no identifiable cause) or secondary (an underlying disease is identifiable). This distinction will determine if further evaluation should be performed to find a potentially reversible cause of hypertension, such as renovascular hypertension origin.

Results: The measurement of blood pressure is mandatory from age 3 in routine and emergency room visits. It should be measured early in newborns in the Neonatal Intensive Care Unit, in patients with congenital urinary tract malformations or abnormalities. Urine examination I and situations with increased intracranial pressure. Moreover, if personal or family history of kidney disease, solid organ and/or bone marrow transplant, cancer, and use of medications that increase BP (beta2stimulants). 
Conclusion: It became clear that hypertension begins in childhood and adolescence, and contributes to early development of cardiovascular disease (CVD).

Keywords: hypertension, childhood, cardiovascular disease

\section{Referências}

1. National High Blood Pressure Education Program Working Group on High Blood Pressure in Children and Adolescents. The Fourth Report on the Diagnosis, Evaluation, and Treatment of High Blood Pressure in Children and Adolescents. Pediatrics 2004; 114: 555-576.

2. Mitsnefes MM. Hypertension in children and adolescents. Pediatr Clin N Am 2006; 53: 493-512.

3. Update on the 1987 Task Force Report on High Blood Pressure in Children and Adolescents: a working group report from the National High Blood Pressure Education Program. National High Blood Pressure Education Program Working Group on Hypertension Control in Children and Adolescents. Pediatrics 1996; 98 : 649-658..

4. Sorof JM, Portman RJ. Ambulatory blood pressure measurements. Curr Opin Pediatr 2001; 13: 133-137.

5. Simckes AM, Srivastava T, Alon US. Ambulatory blood pressure monitoring in children and adolescents. Clin Pediatr (Phila) 2002; 41: 549-564.

6. Lurbe E, Sorof JM, Daniels SR. Clinical and research aspects of ambulatory blood pressure monitoring in children. J Pediatr 2004; 144: 7-16.

7. Soergel M, Kirschstein M, Busch C, Danne T. Gellermann J. Holl R. Krull F. Reichert H. Reusz GS. Rascher W. Oscillometric twenty-four-hour ambulatory blood pressure values in healthy children and adolescents: a multicenter trial including 1141 subjects. J Pediatr 1997; 130: 178-184.

8. Koch VH. Aspectos diagnósticos e terapêuticos atuais da hipertensão arterial na criança e no adolescente, com ênfase no "Fourth Reporto $n$ the Diagnosis, Evaluation, and Treatment of High Blood Pressure in Children and Adolescents" de 2004. J Brás Nefrol 2005; 27: 84-92. 
9. Sociedade Brasileira de Cardiologia- SBC, Sociedade brasileira de Hipertensão -SBH, Sociedade brasileira de Nefrologia- SBN. V Diretrizes Brasileiras de Hipertensão Arterial. 2006: 7-16.

10. Salgado CXM, Carvalhaes JTA. Hipertensão arterial na infância. J Pediatr (Rio J) 2003; 79 (Supl 1): S115-S124.

11. Brewer ED. Evaluation of hypertension in childhood diseases. In Avner ED, Harmon WE, Niaudet P editors. Pediatric Nephrology. 5 th ed. Philadelphia: Lippincott Williams \& Wilkins; 2004. p 1179-97.

12. Sinaiko AR. Current concept: Hypertension in children. N Engl J Med 1996; 335: 1968-1973.

13. National Heart, Lung and Blood Institute, Bethesda, Maryland. Report of the Second Task Force on Blood Pressure Control in Children. Pediatrics 1987; 79: 1-25. 